\title{
A note on generalized Hall planes
}

\section{N.L. Johnson}

\begin{abstract}
We prove that if $\pi$ is a generalized Hall plane of odd order with associated Baer subplane $\pi_{0}$ then $\pi$ is a Hall plane if and only if there is a collineation $\sigma$ of $\pi$ such that $\pi_{0} \sigma \cap \pi_{0}$ is an affine point.
\end{abstract}

Generalized Hall planes were introduced by Kirkpatrick in [4]. The author [2] pointed out that such planes of odd order are derivable from translation planes of semi-translation class $1-3 a$. Ostrom further observed that derivable translation planes of class $1-3 a$ are semifield planes.

Kirkpatrick [5] has characterized the Hall planes of odd order as those generalized Hall planes with associated Baer subplane $\pi_{0}$ such that every affine line of $\pi_{0}$ is the axis of an involutory homology fixing $\pi_{0}$.

This note shows that an easy characterization is also available by assuming there is a collineation group which does not fix $\pi_{0}$.

We shall assume the reader is familiar with "derivation" as developed by Ostrom in [6].

THEOREM. An affine translation plane $\pi$ of odd square order is a Hall plane if and only if there is a Baer subplane $\pi_{0}$ of $\pi$ and a group of collineations $G$ such that

(i) $G$ contains a subgroup $B$ which is transitive on $l_{\infty}-l_{\infty} \cap \pi_{0}$

Received 21 October 1972. This work was partially supported by a Grant Prom the National Science Foundation of the USA. 
and fixes $\pi_{0}$ pointwise, and

(ii) there is an element $\sigma$ of $G$ such that $\pi_{0} \sigma \cap \pi_{0}$ is an affine point.

Proof. Assune $\pi$ satisfies (i) and (ii). By [4], [2], and Ostrom's observation, $\pi$ is derivable from a semifield plane $\bar{\pi}$ where coordinates are chosen so that $\pi_{0}$ in $\pi$ is $\{(x, y) \mid x=0\}$ in $\bar{\pi}$ and $\bar{\pi}$ is a dual translation plane with respect to $(\infty)$.

Let $\sigma \in G$ such that $\pi_{0} \sigma \cap \pi_{0}$ is an affine point. By (2.6) [3], the full collineation group of $\pi$ is the group "inherited" from $\vec{\pi}$. That is, $\sigma$ is also a collineation of $\bar{\pi}$.

The points of $\pi$ and $\bar{\pi}$ are identical so $(x=0) \sigma n(x=0)$ is an affine point of $\bar{\pi}$. Hence $\sigma$ must move $(\infty)$. It is well known that in this situation. $\bar{\pi}$ must be desarguesian so that $\pi$ is a Hall plane by [1].

Conversely, if $\pi$ is a Hall plane then $\bar{\pi}$ is desarguesian and the $((\infty), x=0)-,((0), x=0)-,((0), y=0)$-central collineations of $\bar{\pi}$ which induce collinations of $\pi$ satisfy $(i)$ and $(i i)$.

\section{References}

[1] A.A. Albert, "The finite planes of Ostrom", Bol. Sor. Mat. Mexicana $11(1966), 1-13$.

[2] N.L. Johnson, "A characterization of generalized Hall planes", Bull. Austral. Math. Soc. 6 (1972), 61-67.

[3] N.L. Johnson, "Collineation groups of derived semifield planes", (to appear).

[4] P.B. KI rkpatrick, "Generalization of Hall planes of odd order", Bulz. Austral. Math. Soc. 4 (1971), 205-209.

[5] P.B. Kirkpatrick, "A characterization of the Hall planes of odd order", Bull. Austral. Math. Soc. 6 (1972), 407-415. 
[6] T.G. Ostrom, "Vector spaces and construction of finite projective planes", Arch. Math. 19 (1968), 1-25.

Department of Mathematics,

University of lowa,

lowa City,

lowa,

USA. 